\title{
CT-based multi-phase Radiomic models for differentiating clear cell renal cell carcinoma
}

\author{
Menglin Chen ${ }^{1,2 \dagger}$, Fu Yin ${ }^{3 \dagger}$, Yuanmeng Yu ${ }^{4}$, Haijie Zhang ${ }^{5^{*}}$ and Ge Wen ${ }^{1 *}$ (D)
}

\begin{abstract}
Background: The aim of the study is to compare the diagnostic value of models that based on a set of CT texture and non-texture features for differentiating clear cell renal cell carcinomas(ccRCCs) from non-clear cell renal cell carcinomas(non-ccRCCs).

Methods: A total of 197 pathologically proven renal tumors were divided into $\operatorname{ccRCC}(n=143)$ and non-ccRCC $(n=$ 54) groups. The 43 non-texture features and 296 texture features that extracted from the 3D volume tumor tissue were assessed for each tumor at both Non-contrast Phase, NCP; Corticomedullary Phase, CMP; Nephrographic Phase, NP and Excretory Phase, EP. Texture-score were calculated by the Least Absolute Shrinkage and Selection Operator (LASSO) to screen the most valuable texture features. Model 1 contains the three most distinctive nontexture features with $p<0.001$, Model 2 contains texture scores, and Model 3 contains the above two types of features.
\end{abstract}

Results: The three models shown good discrimination of the cCRCC from non-CCRCC in NCP, CMP, NP, and EP. The area under receiver operating characteristic curve (AUC)values of the Model 1, Model 2, and Model 3 in differentiating the two groups were $0.748-0.823,0.776-0.887$ and $0.864-0.900$, respectively. The difference in AUC between every two of the three Models was statistically significant $(p<0.001)$.

Conclusions: The predictive efficacy of ccRCC was significantly improved by combining non-texture features and texture features to construct a combined diagnostic model, which could provide a reliable basis for clinical treatment options.

Keywords: Clear cell renal cell carcinoma, Radiomics, Improved enhanced parameters, LASSO regression

\section{Background}

Renal cell carcinoma (RCC), as the seventh most common malignant tumors in humans, categorized generally into two major groups: clear cell renal cell carcinoma

\footnotetext{
* Correspondence: zhanghaijie1984@163.com; m13360022166@163.com ${ }^{+}$Menglin Chen and Fu Yin contributed equally to this work.

${ }^{5}$ Department of Radiology, Shenzhen Second People's Hospital, No.3002, West Sungang Road, Futian District, Shenzhen 518052, China

'Medical Imaging teaching and research office, Nanfang hospital, Southern Medical University, No.1838 Guangzhoudadao Avenue north, Guangzhou 510515, Guangdong, China

Full list of author information is available at the end of the article
}

(ccRCC) and non-clear cell renal cell carcinoma (nonccRCC) [1]. Non-ccRCC mainly includes papillary RCC (pRCC) and chromophobe RCC (chRCC) [2]. ccRCC and non-ccRCC have significantly different prognoses, genetic expression patterns, and therapeutic approaches, and ccRCC has much worse prognosis and accounts for $94 \%$ of metastatic RCC $[1,2]$. Furthermore, ccRCC and non-ccRCC have different responses to molecularly targeted therapies, especially in advanced and metastatic RCCs $[3,4]$. Therefore, accurate classification of ccRCC

(c) The Author(s). 2021 Open Access This article is licensed under a Creative Commons Attribution 4.0 International License, which permits use, sharing, adaptation, distribution and reproduction in any medium or format, as long as you give appropriate credit to the original author(s) and the source, provide a link to the Creative Commons licence, and indicate if changes were made. The images or other third party material in this article are included in the article's Creative Commons licence, unless indicated otherwise in a credit line to the material. If material is not included in the article's Creative Commons licence and your intended use is not permitted by statutory regulation or exceeds the permitted use, you will need to obtain permission directly from the copyright holder. To view a copy of this licence, visit http://creativecommons.org/licenses/by/4.0/ The Creative Commons Public Domain Dedication waiver (http://creativecommons.org/publicdomain/zero/1.0/) applies to the data made available in this article, unless otherwise stated in a credit line to the data. 
and non-ccRCC before surgery or treatment has great clinical significance.

Percutaneous renal biopsy is the gold standard for the diagnosis of pathological characteristics within renal mass, but it is an invasive examination, and there may be intolerance for some elderly patients with weak constitution. Therefore, non-invasive imaging method is of great value.

Due to individual patient and technical factors, normalizing the lesion attenuation and the reference standard is essential to normalize for the iodine load and variations in relative lesion attenuation. Previous studies have shown that computed tomography $(\mathrm{CT})$ and Magnetic resonance (MR) based imaging methods contribute to the differentiation of ccRCC from other subtypes [58], but this technique is subjective and the diagnostic accuracy still depends on the doctor's clinical experience.

In recent years, artificial intelligence methods using Radiomic Features (RFs) analysis has gradually attracted increased attention. RFs characterize microscale information that cannot be recognized by naked human eyes, such as texture features $[9,10]$. The method of RFs analysis can extract and analyze tumor information hidden within conventional medical imaging in a high-flux way to provide radiologists with more accurate image diagnostic information and decision support in the clinic regardless of the experience of clinicians [9-11]. Previous studies have demonstrated that RFs could potentially be used in cancer classification and survival prediction for different cancers such as colorectal cancer [12, 13]. Compared with those high incidence and high mortality rate tumors, texture features are less used in renal tumors, and there are still many spaces for improvement. To our knowledge, some studies did not include the entire 3D tumor tissue or four-phase images for analysis, which may lead to the loss of some tumor biological information [14, 15]. In addition, most studies have focused on the differentiation between renal malignancy and benign renal tumors, and relatively few studies have focused on the differentiation between ccRCC and nonccRCC $[16,17]$. Furthermore, few studies have incorporated the $\mathrm{CT}$ texture and non-texture features to construct a radiomic model.

In summary, the purpose of this study is to compare the predictive models that based on a set of CT texture and non-texture features for differentiating ccRCC from non-ccRCC.

\section{Methods}

\section{Patients}

This study was approved by the Ethics Committee of our hospital (approval number: NFEC-2018-104), and the patients' informed consent were waived. The 197 patients in this study were obtained from the picture archiving and communication system (PACS) of our hospital from January 2013 to December 2018.

The inclusion criteria were as follows: (1) patients with no previous treatment before CT examination;(2) CT examination with no significant image noise or significant artefacts; (3) CT examination with four phases (the non-contrast phase, NCP; the corticomedullary phase, CMP; the nephrographic phase, NP and the excretory phase, EP). The exclusion criteria were as follows: (1) RCC with most or all of the cysts features;(2) patients with two or more lesions in unilateral or bilateral kidneys to avoid potential clustering effects; (3) RCC with multiple pathologic types of tumors to avoid different pathological types affect each other.

Of these cases, including 143 ccRCCs, 25 pRCCs and 29 chRCCs, 81 patients' final pathological diagnosis were acquired from partial nephrectomy,109 from radical nephrectomy and 7 from biopsy. There were no differences in patient age or sex or maximum tumor diameter comparing ccRCCs (mean age \pm standard deviation (SD), $53.202 \pm 13.086$ years; mean diameter \pm SD, $5.283 \pm$ $2.602 \mathrm{~cm} ; 92$ men and 51 women; 62 tumor located in the right kidney, and 81 in the left) with non-ccRCCs (52.804 \pm 12.857 years; $4.929 \pm 2.615 \mathrm{~cm} ; 31$ men and 23 women; 31 tumor located in the right kidney, and 23 in the left) tumors $(p=0.691,0.373,0.323$ and 0.923 respectively). Demographic and clinical characteristics of patients with ccRCC and non-ccRCC is summarized in Table 1.

\section{Acquisition of $\mathrm{CT}$ images}

The data were collected from a 64-multidetector spiral CT scanner (Somatom Definition CT, siemens Medical Solutions) and a 256-multidetector spiral CT scanner (Brilliance iCT, Philips Medical Systems). The patients were in supine position, and the scanning range was from the phrenic top to the lower pole of both kidneys during breath holding. The nonionic iodine contrast agent (Iohexol, GE, American; Ultravist, Bayer, Germany) was injected by high pressure injector at a dose of $2 \mathrm{ml} / \mathrm{kg}$ and a speed of $2.5 \mathrm{ml} / \mathrm{s}$. All the studies involved at least four-phase scanning, including CMP, PP, EP,which were started at 30-35th,60-70th,190 200th second after the contrast agent was injected into the antecubital vein. The scanning parameters were as follows: tube voltage $120 \mathrm{kV}$, tube current $150-320 \mathrm{~mA}$, thickness of scan layer and the interlayer spacing $5 \mathrm{~mm}$, field of view (FOV) $360 \mathrm{~mm}$, a matrix of $512 \times 512$.

Image normalisation and grey level discretisation procedures were done to minimise inter-scanner effects and variations. We used the \pm 3 sigma technique to normalized CT image signal intensities $[18,19]$. Follow this, the grey level discretisation was done by assigning a grey level range between 1 and $2 \mathrm{k}$, where $\mathrm{k}$ is the bits/pixel 
Table 1 Demographic and clinical characteristics of patients with CCRCC and non-CCRCC

\begin{tabular}{llll}
\hline characteristic & ccRCC & non-ccRCC & P Value \\
\hline Number & 143 & 54 \\
Gender & & \\
$\quad$ Male & 92 & 31 \\
$\quad$ Female & 51 & 23 & 0.323 \\
Age (mean \pm SD, years) & $53.202 \pm 13.086$ & $52.804 \pm 12.857$ \\
Maximum diameter (mean \pm SD, cm) & $5.283 \pm 2.602$ & $4.929 \pm 2.615$ & \\
Tumor location & & 31 & 0.691 \\
$\quad$ Right & 62 & 23 & 0.923 \\
Left & 81 & 2373 \\
\hline
\end{tabular}

[20]. The k-value was chosen to be 6 for co-occurrence matrix features and run-length matrix features, 4 for gradient features, and 8 for wavelet features.

\section{Construction of non-texture features model Image analysis}

This part of the data set consists of CT values of lesions. Two region of interests (ROIs) of approximately 0.5-1 $\mathrm{cm}^{2}$ was manually placed by two radiologists (5 and 12 years of experience) on the most avidly enhancing part of a heterogeneously enhancing lesion or in the center of a homogeneously enhancing lesion on the largest area of lesion. At the same time, necrosis, blood vessels and calcification components should be avoided. In addition, the corresponding position and size of each ROI are consistent in the four phase CT images. Finally, the lesion attenuation value in each phase was obtained by calculating the average attenuation value of the two ROIs. The attenuation value of the renal cortex and aorta were also measured as references to indicate the iodine load (Fig. 1). The enhancement values were calculated with specific formulas in Table 2 .

\section{Establishment of non-texture features model}

The t-test or Mann-Whitney $U$ test was performed to compare the magnitude of the above enhancement parameters of ccRCCs with non-ccRCCs in each of the four phases. A receiver operating characteristic curve (ROC) and the area under the curve (AUC) was used to evaluate the diagnostic performance of the enhanced parameters measurements. The three most discriminative parameters with $P<0.001$ in each phase were then
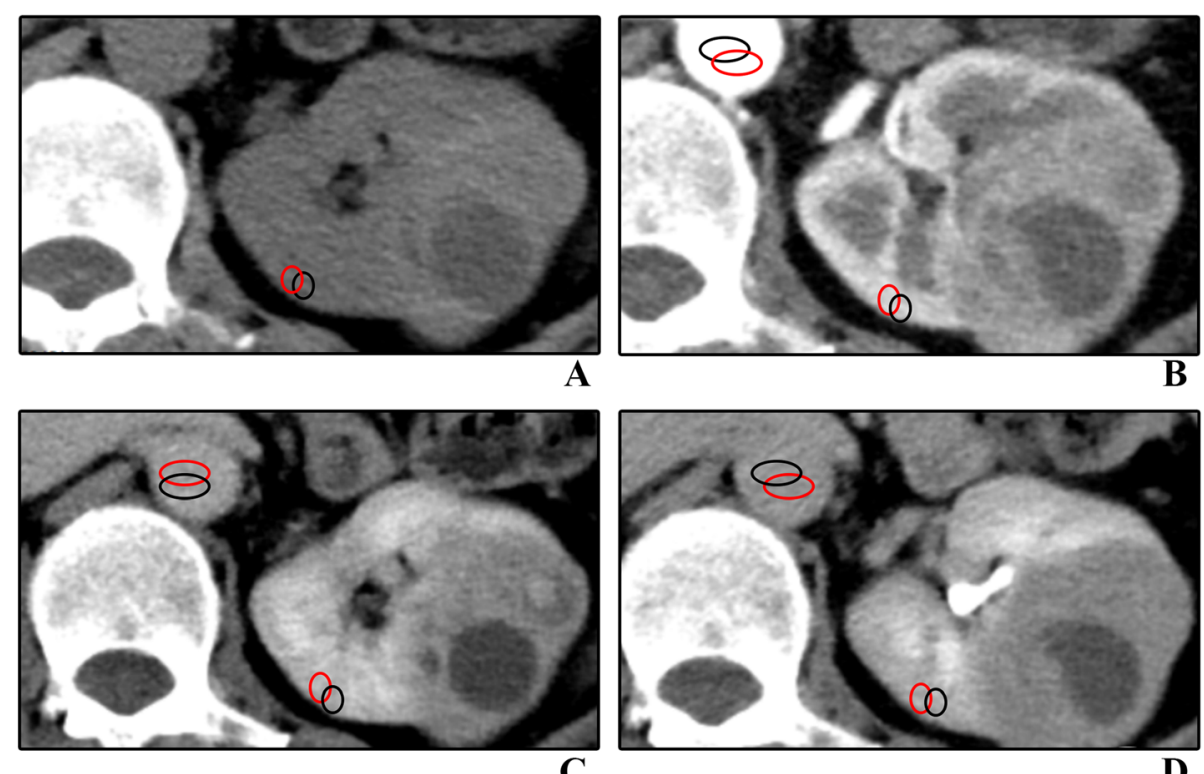

Fig. 1 57-year-old woman with clear cell renal cell carcinoma. The red and black circles represent ROls outlined by two radiologists including cortex ROI and aortic ROI, and the corresponding positions of ROls in the four-phase images are the same. A, Unenhanced image. B, Corticomedullary phase image. C, Nephrographic phase image. D, Excretory phase image 
Table 2 Enhancement parameters of the lesion and its formulas

\begin{tabular}{|c|c|c|}
\hline Enhancement parameters & Formulas & References \\
\hline Lesion Attenuation Value & - & \\
\hline Absolute Deenhancement & $T_{3}-T_{2} ; T_{4}-T_{3}$ & [21] \\
\hline Absolute Enhancement & $T_{2 / 3 / 4}-T_{1}$ & [21] \\
\hline Percentage Enhancement Ratio of Absolute Enhancement & $\left(T_{2 / 3 / 4}-T_{1}\right) / T_{1}$ & \\
\hline Percentage Enhancement Ratio of renal cortex & $C_{1 / 2 / 3 / 4} / T_{1 / 2 / 3 / 4}$ & [22] \\
\hline Percentage Enhancement Ratio of aorta & $T_{2 / 3 / 4} / A_{2 / 3 / 4}$ & [2] \\
\hline Relative Attenuation of renal cortex & $\left(T_{1 / 2 / 3 / 4}-C_{1 / 2 / 3 / 4}\right) / C_{1 / 2 / 3 / 4}$ & [21] \\
\hline Relative Attenuation of aorta & $\left(A_{2 / 3 / 4}-T_{2 / 3 / 4}\right) / A_{2 / 3 / 4}$ & \\
\hline Enhancement Change of renal cortex & $C_{1 / 2 / 3 / 4^{-}} T_{1 / 2 / 3 / 4}$ & [22] \\
\hline Enhancement Change of aorta & $\mathrm{A}_{2 / 3 / 4}-\mathrm{T}_{2 / 3 / 4}$ & \\
\hline Relative Enhancement Value of the Tumor $\left(\mathrm{rTEV}_{2 / 3 / 4}\right)$ & $\left(T_{2 / 3 / 4}-T_{1}\right) /\left(C_{2 / 3 / 4}-C_{1}\right)$ & [2] \\
\hline Corrected $\mathrm{CT}$ values relative to aorta & $\left(\mathrm{Sa} / \mathrm{A}_{2 / 3 / 4}\right) * \mathrm{~T}_{2 / 3 / 4}$ & \\
\hline Corrected $C T$ values relative to renal cortex & $\left(\mathrm{AC} / \mathrm{C}_{1 / 2 / 3 / 4}\right) * \mathrm{~T}_{1 / 2 / 3 / 4}$ & \\
\hline
\end{tabular}

Note: $\mathrm{T} 1, \mathrm{~T} 2, \mathrm{~T} 3$ and $\mathrm{T} 4$ represent the $\mathrm{CT}$ values of the tumors in unenhanced phase, corticomedullary phase, nephrographic phase, excretory phase respectively.C1, C2, C3 and C4 represent the CT values of normal renal cortex adjacent to the tumor in unenhanced phase, corticomedullary phase, nephrographic phase, excretory phase respectively. A2, A3 and A4 represent the CT values of the aorta in corticomedullary phase, nephrographic phase, excretory phase respectively. Sa represents the mean $\mathrm{CT}$ values of the corresponding layers of aorta in corticomedullary phase, nephrographic phase, and excretory phase respectively. Ac represents the mean CT value of the normal renal cortex adjacent to the tumor in unenhanced phase, corticomedullary phase, nephrographic phase, and excretory phase respectively

entered as predictors in logistic regression models, and ROC curves were computed. The t-test or MannWhitney $U$ test was performed to compare differences between two groups. The AUC was used to determine the threshold with the highest accuracy in discriminating ccRCC from non-ccRCC. For each threshold level, sensitivity, specificity, positive predictive value (PPV), negative predictive value (NPV), and accuracy were calculated. Comparisons among AUCs between every two model were evaluated according to DeLong test.
Matthews correlation coefficient (MCC) was also used to overcome the class imbalance issue.

\section{Construction of texture features model Image segmentation}

Axial images of four phases (NCP, CMP, NP, and EP) with a layer thickness of $5 \mathrm{~mm}$ were included in this study. These original images were manually segmented by two radiologists in a laptop equipped with ITK-SNAP software (www.itk-snap.org). The entire 3D tumor tissue
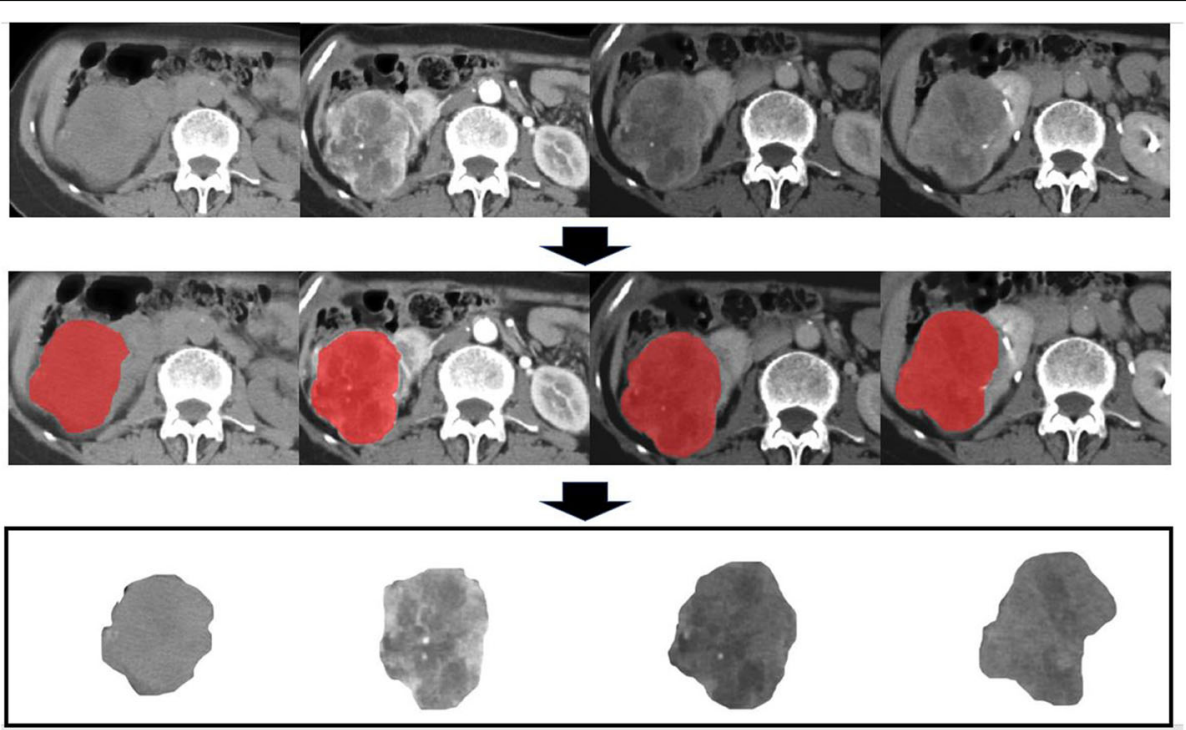

Fig. 2 Graph shows the process of obtaining tumor tissue from original images by manual segmentation in unenhanced, corticomedullary, nephrographic, and excretory phase from left to right 
was segmented with margin shrinkage of $2 \mathrm{~mm}$ from the lesion contour to avoid the inclusion of peripheral fat and normal renal tissue [23]. The specific image segmentation process is shown in Fig. 2.

\section{Texture features extraction}

Texture features were extracted using PyRadiomics [11]. The segmented images were loaded and pre-processed, and over-sampling is used in order to solve the problem of data imbalance between ccRCCs and non-ccRCCs. Then texture features were extracted using the categories of gray level cooccurrence matrix, (GLCM), gray level run length matrix, (GLRLM), gray level size zone matrix, (GLSZM), neighbouring gray tone difference matrix, (NGTDM) and gray level dependence matrix, (GLDM).

\section{Establishment of texture features model}

We used the least absolute shrinkage and selection operator (LASSO) to select the most valuable texture features and calculated the texture score (Texture-score) for each patient via a linear combination of the selected features weighted by their corresponding coefficients. Mann-Whitney U test was performed to compare differences between texture scores of ccRCC and non-ccRCC group. The AUC was used to determine the threshold with the highest accuracy in discriminating ccRCC from non-ccRCC. For each threshold level, sensitivity, specificity, PPV, NPV, and accuracy were calculated. Comparisons among AUCs between every two model were evaluated according to DeLong test. MCC was also used to overcome the class imbalance issue.

\section{Construction of combined diagnostic model}

On the basis of non-texture features (Model 1), and of Texture-score (Model 2), a logistic regression with those above variables was performed to build a combined diagnostic model (Model 3). For each threshold level, sensitivity, specificity, PPV, NPV, and accuracy were also calculated. Comparisons among AUCs between every two model were evaluated according to DeLong test. MCC was also used to overcome the class imbalance issue.

\section{Statistical analysis}

All analyses were performed by using SPSS (IBM SPSS Statistics for Windows, Version 20.0. Armonk, NY: IBM Corp) and $\mathrm{R}$ version 3.1.0 ( $\mathrm{R}$ Foundation). Although $\mathrm{P}<$ 0.05 was considered to indicate a significant difference, we used a stepwise Holm-Bonferroni procedure to counteract the potential for type I errors arising from multiple comparisons. The 43 features were first ranked in ascending order of $P$ value; if the first $P$ value was less than $0.05 / 43$, we then compared the second $\mathrm{P}$ value to $0.05 /(43-1)$, and we continued this stepwise comparison until no further $P$ values indicated a significant difference.

A synthetic minority oversampling technique (SMOTE) [24] was adopted by sample generation of minority group from joint weighting of optimal features in order to tackle the adverse impact of the imbalance dataset in this study (143:54) on the performance of texture features. The synthetic cases will have attributes with values similar to the existing cases and not merely replications in order to increase the representation of the non-ccRCC group in the dataset while reflecting the structure of the original cases $[25,26]$.

Re-proposed by Baldi and colleagues [27] in 2000, MCC is a standard performance metric for machine learning, and was usually used to overcome the class imbalance issue through its mathematical properties invariances for class swapping. MCC now has become a successful indicator with a natural extension to the multiclass case [28]. Besides, the $F_{1}$ measure is widely used in most application areas of machine learning, not only in the binary scenario, but also in multiclass cases.

$$
\begin{aligned}
& M C C=\frac{T P * T N-F P * F N}{\sqrt{(T P+F P) *(T P+F N) *(T N+F P) *(T N+F N)}} \\
& F 1 \text { score }=\frac{2 \mathrm{TP}}{2 T P+F P+F N}
\end{aligned}
$$

$\mathrm{TP}=$ Ture positives. $\mathrm{TN}=$ Ture negatives. $\mathrm{FN}=$ False negatives. $\mathrm{FP}=$ False positives.

\section{Results}

Construction of non-texture features model (Model1)

There were $0,12,13$, and 8 non-texture features statistically significant for distinguishing ccRCC from nonccRCC in NCP, CMP, NP, and EP respectively. In those non-texture features, most of the improved enhancement parameters had higher diagnostic efficacy than directly measured lesion attenuation value, and the specific results were shown in Table 3.

Three most discriminative parameters in each phase were then entered as predictors in logistic regression models to construct three diagnostic models in CMP, NP and EP phase respectively. Since all the variables of the NCP had no significant statistical significance for the identification of ccRCC and non-ccRCC, the diagnostic model of the NCP was not established. Finally, three different non-texture features models were constructed.

The Model 1(CMP) incorporated the three most common features identified in CMP (Percentage Enhancement Ratio of Absolute Enhancement, Relative Attenuation of renal cortex, and rTEV). The Model $1(\mathrm{NP})$ incorporated the three most common features identified in NP (Percentage Enhancement Ratio of Absolute Enhancement, Relative Attenuation of aorta and 
Table 3 Non-texture features of ccRCC and non-ccRCC

\begin{tabular}{|c|c|c|c|c|}
\hline & ccRCC & non-ccRCC & $P$ value & AUC \\
\hline \multicolumn{5}{|l|}{ NCP } \\
\hline Lesion Attenuation Value & $33.996 \pm 9.443$ & $35.843 \pm 7.821$ & 0.109 & 0.574 \\
\hline Relative Attenuation of renal cortex & $0.075 \pm 0.320$ & $0.105 \pm 0.297$ & 0.610 & 0.624 \\
\hline Percentage Enhancement Ratio of renal cortex & $1.035 \pm 0.387$ & $0.984 \pm 0.288$ & 0.528 & 0.529 \\
\hline Enhancement Change of renal cortex & $-1.862 \pm 9.447$ & $-2.567 \pm 9.514$ & 0.640 & 0.522 \\
\hline Corrected $\mathrm{CT}$ values relative to renal cortex & $34.506 \pm 10.119$ & $34.551 \pm 9.685$ & 0.213 & 0.658 \\
\hline \multicolumn{5}{|l|}{ CMP } \\
\hline Lesion Attenuation Value & $124.394 \pm 47.847$ & 78.494士34.898 & $<0.001^{*}$ & 0.779 \\
\hline Absolute Enhancement & $90.403 \pm 49.572$ & $42.661 \pm 31.789$ & $<0.001^{*}$ & 0.784 \\
\hline Percentage Enhancement Ratio of Absolute Enhancement & $3.010 \pm 2.283$ & $1.174 \pm 0.850$ & $<0.001^{*}$ & 0.803 \\
\hline Relative Attenuation of renal cortex & $-0.972 \pm 0.371$ & $-0.456 \pm 0.241$ & $<0.001^{*}$ & 0.809 \\
\hline Percentage Enhancement Ratio of renal cortex & $1.396 \pm 0.846$ & $2.368 \pm 1.263$ & $<0.001^{*}$ & 0.799 \\
\hline Enhancement Change of renal cortex & $17.664 \pm 47.849$ & $69.795 \pm 43.817$ & $<0.001^{*}$ & 0.780 \\
\hline rTEV & $0.886 \pm 0.738$ & $0.409 \pm 0.324$ & $<0.001^{*}$ & 0.800 \\
\hline Corrected $C T$ values relative to renal cortex & $128.291 \pm 52.369$ & $81.609 \pm 36.276$ & $<0.001^{*}$ & 0.787 \\
\hline Corrected $C T$ values relative to aorta & $127.631 \pm 45.998$ & $81.623 \pm 37.425$ & $<0.001^{*}$ & 0.787 \\
\hline Percentage Enhancement Ratio of aorta & $0.439 \pm 0.153$ & $0.287 \pm 0.138$ & $<0.001^{*}$ & 0.777 \\
\hline Relative Attenuation of aorta & $0.571 \pm 0.157$ & $0.722 \pm 0.135$ & $<0.001^{*}$ & 0.777 \\
\hline Enhancement Change of aorta & 174.474士73.398 & $215.335 \pm 85.426$ & $0.004^{*}$ & 0.733 \\
\hline \multicolumn{5}{|l|}{ NP } \\
\hline Lesion Attenuation Value & $91.727 \pm 22.870$ & $73.092 \pm 23.618$ & $<0.001^{*}$ & 0.725 \\
\hline Absolute Enhancement & $57.735 \pm 23.909$ & $37.259 \pm 21.359$ & $<0.001^{*}$ & 0.755 \\
\hline Absolute Deenhancement & $-32.674 \pm 34.765$ & $-5.400 \pm 20.700$ & $<0.001^{*}$ & 0.742 \\
\hline Percentage Enhancement Ratio of Absolute Enhancement & 1.913士1.213 & 1.074士0.626 & $<0.001^{*}$ & 0.756 \\
\hline Relative Attenuation of renal cortex & $-0.322 \pm 0.187$ & $-0.431 \pm 0.181$ & $<0.001^{*}$ & 0.712 \\
\hline Percentage Enhancement Ratio of renal cortex & $1.627 \pm 0.623$ & $2.366 \pm 1.263$ & $<0.001^{*}$ & 0.699 \\
\hline Enhancement Change of renal cortex & 45.249士22.876 & $59.712 \pm 28.872$ & $0.001^{*}$ & 0.660 \\
\hline rTEV & $0.575 \pm 0.281$ & $0.393 \pm 0.227$ & $<0.001^{*}$ & 0.729 \\
\hline Corrected $C T$ values relative to renal cortex & $93.596 \pm 24.267$ & $74.648 \pm 24.734$ & $<0.001^{*}$ & 0.735 \\
\hline Corrected $C T$ values relative to aorta & $91.923 \pm 18.694$ & $73.195 \pm 21.801$ & $<0.001^{*}$ & 0.751 \\
\hline Percentage Enhancement Ratio of aorta & $0.753 \pm 0.150$ & $0.593 \pm 0.188$ & $<0.001^{*}$ & 0.761 \\
\hline Relative Attenuation of aorta & $0.258 \pm 0.154$ & $0.419 \pm 0.184$ & $<0.001^{*}$ & 0.761 \\
\hline Enhancement Change of aorta & $30.156 \pm 19.672$ & $50.440 \pm 23.580$ & $<0.001^{*}$ & 0.750 \\
\hline \multicolumn{5}{|l|}{ EP } \\
\hline Lesion Attenuation Value & 72.978士16.014 & 63.385士16.952 & $<0.001^{*}$ & 0.670 \\
\hline Absolute Enhancement & 38.995士16.019 & $27.550 \pm 14.126$ & $<0.001^{*}$ & 0.730 \\
\hline Absolute Deenhancement & $-18.759 \pm 12.282$ & $-9.701 \pm 11.864$ & $<0.001^{*}$ & 0.704 \\
\hline Percentage Enhancement Ratio of Absolute Enhancement & $1.294 \pm 0.806$ & $0.793 \pm 0.418$ & 0.077 & 0.602 \\
\hline Relative Attenuation of renal cortex & $-0.388 \pm 0.134$ & $-0.468 \pm 0.134$ & 0.341 & 0.555 \\
\hline Percentage Enhancement Ratio of renal cortex & $1.724 \pm 0.550$ & $2.034 \pm 0.600$ & 0.678 & 0.524 \\
\hline Enhancement Change of renal cortex & $45.673 \pm 16.017$ & $56.313 \pm 21.001$ & 0.789 & 0.515 \\
\hline rTEV & $0.467 \pm 0.191$ & $0.339 \pm 0.156$ & 0.143 & 0.584 \\
\hline Corrected $\mathrm{C} T$ values relative to renal cortex & 74.002士15.976 & $64.228 \pm 15.503$ & $0.011^{*}$ & 0.647 \\
\hline Corrected $C T$ values relative to aorta & $73.050 \pm 12.505$ & $63.274 \pm 13.974$ & $0.016^{*}$ & 0.639 \\
\hline
\end{tabular}


Table 3 Non-texture features of ccRCC and non-ccRCC (Continued)

\begin{tabular}{|c|c|c|c|c|}
\hline & ccRCC & non-ccRCC & $P$ value & AUC \\
\hline Percentage Enhancement Ratio of aorta & $0.784 \pm 0.133$ & $0.676 \pm 0.152$ & $<0.001^{*}$ & 0.729 \\
\hline Relative Attenuation of aorta & $0.222 \pm 0.137$ & $0.331 \pm 0.158$ & $<0.001^{*}$ & 0.729 \\
\hline Enhancement Change of aorta & 20.779士13.349 & $31.112 \pm 14.534$ & $<0.001^{*}$ & 0.718 \\
\hline
\end{tabular}

Note: * represents statistically significant; the non-contrast phase, NCP; the corticomedullary phase, CMP; the nephrographic phase, NP and the excretory phase, EP

Percentage Enhancement Ratio of aorta). The Model $1(E P)$ incorporated the three most common features identified in EP (Percentage Enhancement Ratio of aorta, Absolute Enhancement, and Relative Attenuation of aorta). There were statistically significant differences between the diagnostic models of CMP, NP and EP in distinguishing ccRCC and non-ccRCC $(P<0.001)$.

\section{Performance of model $\mathbf{1}$ for distinguishing ccRCC}

The AUC and MCC value of the CMP was the highest among the three models, reaching $0.823(95 \%$ Confidence interval $(\mathrm{CI}): 0.745-0.901)$, and +0.557 . However, the accuracy and $F_{1}$ score of the NP were the highest, which were 0.812 and 0.844 respectively. The ROC curves results were shown in Fig. 3A.
DeLong test results showed that there was no statistically significant difference between the model in NP and model in $\operatorname{EP}(P=0.120)$. In other words, the AUC value of model in CMP was higher than that of NP and EP $(P=0.032 ; P=0.006)$.

\section{Construction of texture features model (Model2)}

There were 74 features been extracted from every single phase of CT scanning, therefore, the total number of the features extracted was 296 per lesion. Those features were: 23 GLCM features, 16 GLSZM features, 16 GLRL $M$ features, 14 GLDM features, and 5 NGTDM features. The texture features and formulas of the diagnostic Model 2 in each phase were shown in Table 4.

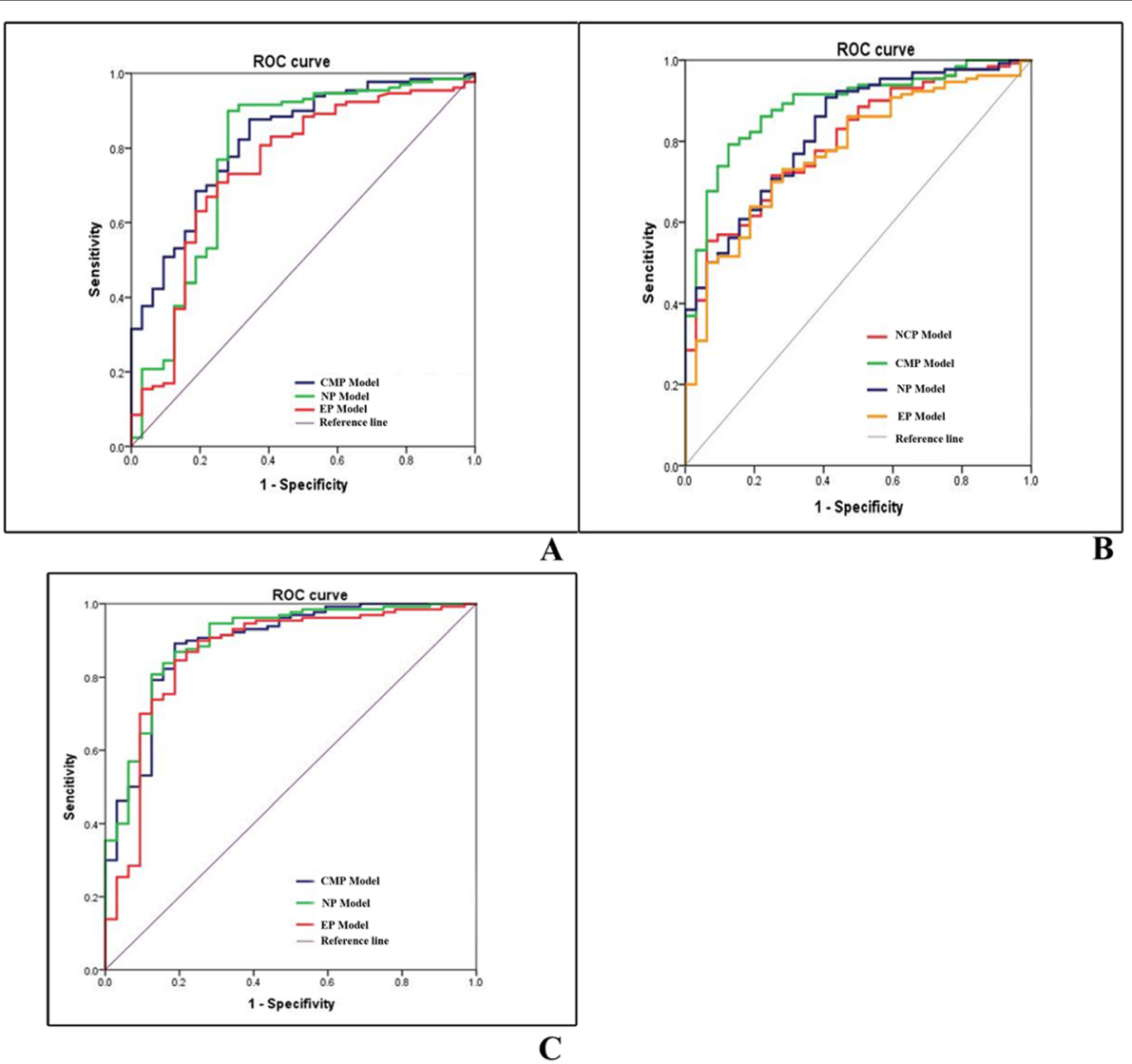

Fig. 3 Graph shows ROC curves for differentiation of ccRCCs and non-ccRCCs on basis of non-texture features(A), texture features(B), and combined featuers(C) 
Table 4 Features and formulas for the identification of cCRCC and non-cCRCC

\begin{tabular}{ll}
\hline Features & Formulas \\
\hline f47: GLCM Maximum Probability & $\max (P(i, j))$ \\
f49: GLCM Joint Energy & $\sum_{i=1}^{N_{g}} \sum_{j=1}^{N_{g}}(P(i, j))^{2}$ \\
f56: GLCM Correlation & $\frac{\sum_{i=1}^{N_{g}} \sum_{j=1}^{N_{g}} P(i, j) j-\mu_{x} \mu_{y}}{\sigma_{x}(i) \sigma_{y}(j)}$ \\
f58: GLCM Sum Entropy & $\sum_{k=2}^{2 N_{g}} P_{x+y}(k) \log _{2}\left(P_{x+y}(k)+\varepsilon\right)$ \\
f94: GLRLM Long Run High Gray Level Emphasis & $\frac{\sum_{i=1}^{N_{g}} \sum_{j=1}^{N_{g}} P(i, j) i^{2} j^{2}}{\sum_{i=1}^{N_{g}} \sum_{j=1}^{N_{g}} P(i, j)}$ \\
f102: GLSZM Gray Level Non Uniformity Normalized & $\frac{\sum_{i=1}^{N_{g}}\left(\sum_{j=1}^{N_{g}} P(i, j)\right)^{2}}{N_{z}^{2}}$ \\
\hline
\end{tabular}

The 296 features were included in the LASSO regression model to screen the most valuable features. It is known that tuning parameter $(\lambda)$ corresponds to various diagnostic characteristic curves. The optimal $\lambda$ selection criteria contain the minimum standard and 1-SE. We found that through preliminary research results the difference in AUC values of the two models selected by the above two criteria were very small. To construct a more concise model, $\lambda$ was selected by 1 -SE standard.

Finally, Texture-scores were obtained by LASSO regression. The significance of each texture parameter and texture-score in distinguishing ccRCC from non-ccRCC is summarized in Table 5 .

\section{Performance of model 2 for distinguishing ccRCC}

Among the four models, the AUC,accuracy,MCC of the Texture -score(CMP) were the highest, which were $0.887,0.809$, and 0.601 respectively; the $F_{1}$ score of the Model 2(NP)reached 0.838. By comparison of AUC values among models at four phases, the results showed that the difference between four phases was not statistically significant $(P=0.07-0.838)$. Specific results are shown in Table. 6 and Fig. 3B.

\section{Construction of combined diagnostic model (Model3)}

The results of the two-class multivariate logistic regression model showed that there were 2 (Texture-score, Enhancement Change of aorta), 4 (Texture-score, Absolute Deenhancement, Corrected CT values relative to aorta, and Enhancement Change of aortia), and 2 (Texture-score, Enhancement Change of aortia) independent factors in distinguishing ccRCC from non-ccRCC in $\mathrm{CMP}, \mathrm{NP}$, and $\mathrm{EP}$, respectively. In addition, our results showed that the Texture-score in three enhanced phases were independent risk factors for ccRCC diagnosis, that is, the higher the value of the Texture-score, the higher the possibility of clinical indication of ccRCC. Among them, the top two features with the strongest correlation were the Texture-score in NP and EP. Non-texture features were protective factors of ccRCC (odds ratio value $<1$ ), and the correlation was far lower than texture features. Specific results were shown in Table 7.

Table 5 Texture-score and texture features at the NCP, CMP, NP and EP to distinguish ccRCC from non-ccRCC

\begin{tabular}{lllll}
\hline Phase & Texture-score and features & ccRCC & non-ccRCC & P value \\
\hline NCP & $f 47$ & $0.513 \pm 0.136$ & $0.625 \pm 0.141$ & $<.001$ \\
& $f 56$ & $0.287 \pm 0.094$ & $0.196 \pm 0.119$ & $<0.001$ \\
& $f 58$ & $1.582 \pm 0.265$ & $1.344 \pm 0.299$ & 0.001 \\
& $f 94$ & $53.046 \pm 37.244$ & $76.842 \pm 56.910$ & 0.026 \\
& Texture -score & $1.704 \pm 0.538$ & $1.115 \pm 0.551$ & $<0.001$ \\
CMP & f49 & $0.095 \pm 0.057$ & $0.193 \pm 0.080$ & $<0.001$ \\
& Texture -score & $1.723 \pm 0.471$ & $0.810 \pm 0.677$ & $<0.001$ \\
NP & f49 & $0.147 \pm 0.064$ & $0.241 \pm 0.095$ & $<0.001$ \\
& f102 & $0.224 \pm 0.056$ & $0.302 \pm 0.086$ & $<0.001$ \\
Tex & T56 & $1.571 \pm 0.287$ & $1.123 \pm 0.415$ & $<0.001$ \\
& f58 & $0.440 \pm 0.146$ & $0.319 \pm 0.133$ & $<0.001$ \\
& Texture -score & $2.335 \pm 0.360$ & $1.994 \pm 0.274$ & $<0.001$ \\
\end{tabular}


Table 6 Diagnostic performance of Model1, Model 2 and Model 3 for distinguishing CCRCC from non-ccRCC

\begin{tabular}{lllllllllll}
\hline Phase & Model & Cut-off value & AUC $(\mathbf{9 5} \% \mathbf{C l})$ & Sensitivity & Specificity & PPV & NPV & Accuracy & MCC & $\mathbf{F}_{\mathbf{1}}$ score \\
\hline CMP & Model1 & 0.639 & $0.823(0.745-0.901)$ & 0.797 & 0.759 & 0.903 & 0.600 & 0.787 & +0.557 & 0.791 \\
& Model 2 & 1.500 & $0.887(0.828-0.945)$ & 0.875 & 0.792 & 0.515 & 0.963 & 0.809 & +0.601 & 0.835 \\
& Model 3 & 0.718 & $0.891(0.824-0.957)$ & 0.892 & 0.812 & 0.953 & 0.656 & 0.877 & +0.637 & 0.936 \\
NP & Model1 & 0.302 & $0.784(0.680-0.889)$ & 0.881 & 0.630 & 0.867 & 0.675 & 0.812 & +0.548 & 0.844 \\
& Model 2 & 1.179 & $0.826(0.752-0.901)$ & 0.908 & 0.594 & 0.869 & 0.507 & 0.803 & +0.598 & 0.838 \\
& Model 3 & 0.853 & $0.900(0.837-0.963)$ & 0.808 & 0.875 & 0.961 & 0.538 & 0.821 & +0.625 & 0.897 \\
EP & Model1 & 0.927 & $0.748(0.647-0.849)$ & 0.664 & 0.759 & 0.883 & 0.462 & 0.690 & +0.297 & 0.718 \\
& Model 2 & 1.362 & $0.776(0.694-0.859)$ & 0.700 & 0.750 & 0.915 & 0.384 & 0.710 & +0.376 & 0.759 \\
& Model 3 & 0.737 & $0.864(0.783-0.945)$ & 0.846 & 0.812 & 0.953 & 0.571 & 0.840 & +0.627 & 0.915 \\
\hline
\end{tabular}

\section{Performance of model 3 for distinguishing ccRCC}

In combined diagnosis model of three phases, the AUC value of the NP was the highest, reaching $0.900(95 \% \mathrm{CI}$ : 0.837-0.963), followed by the CMP and EP, while the MCC and $\mathrm{F}_{1}$ score of the CMP were the highest, which were 0.637 and 0.936 , respectively. The DeLong test showed no significant difference in AUC values between models in CMP, NP and EP $(P=0.085-1)$. The specific results are shown in Table 6 and Fig. $3 \mathrm{C}$.

\section{Comparison of diagnostic efficacy between model 1, model 2 and model 3}

The study showed that the AUC value of the Model 3 was the highest in any of the three enhanced phases, followed by the Model 2, and Model 1. DeLong test results showed that there were significant differences in AUC values between Model 1 and 2, Model 2 and 3, Model 1 and 3 in any of the three enhanced phases $(P<$ $0.001)$.

The diagnostic performance of CMP in Model 1 was the highest when compared to that in NP and EP, and the differences was statistically significant. In both Model 2 and Model 3, there were no statistically significant differences in the detection efficiency of ccRCC between CMP, NP and EP, which suggested that the diagnostic performance in NP and EP were considered to be consistent with that in CMP. The specific results are shown in Table 6 and Fig. 4.

\section{Discussion}

In this study, we compared the diagnostic performance of models that based on a set of CT texture and nontexture features to differentiate ccRCC from non-ccRCC. In summary, we found that the model with both texture features and non-texture features could stratify patients with higher diagnostic performance. Specifically, textural features related to Maximum Probability, Joint Energy, Correlation, Sum Entropy, Long Run High Gray Level Emphasis and Gray Level Non-uniformity Normalized are the strongest predictors of ccRCC.

Previous studies had indicated the value of radiological imaging in differentiating the ccRCC from other subtypes $[22,29,30]$. Despite some positive results, there were similarities in attenuation between the RCC subtype, especially for small renal lesion [31]. Besides, due to the individual differences in heart and kidney function among different patients, there is a certain degree of deviation between the degree of renal tumor enhancement measured artificially and the actual degree of tumor enhancement. By applying mathematical methods to reduce this bias, the enhancement values, such as Absolute Enhancement, and rTEV, would be helpful for improving confidence in diagnosis and facilitating

Table 7 Multivariate analysis results of combined models in the differentiation of ccRCC from non-ccRCC

\begin{tabular}{lllll}
\hline & Independent influencing factor & Regression coefficients & OR value & P value \\
\hline CMP & Texture-score & 2.654 & 14.205 & 0.988 \\
NP & Enhancement Change of aorta & -0.012 & 61.187 & 0.001 \\
& Texture-score & 4.114 & 0.971 & 0.001 \\
& Absolute Deenhancement & -0.003 & 0.901 & 0.01 \\
Corrected CT values relative to aorta & -0.104 & 0.887 & 0.012 \\
EP & Enhancement Change of aorta & -0.120 & 35.489 & 0.002 \\
& Texture-score & 3.569 & 0.928 & $<0.001$ \\
\hline
\end{tabular}




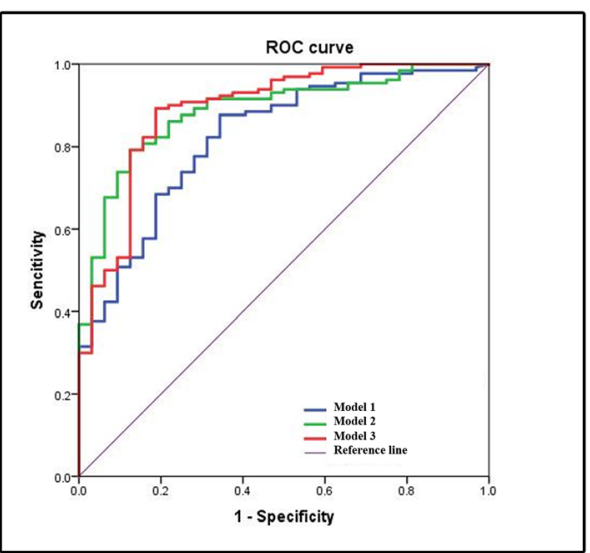

A

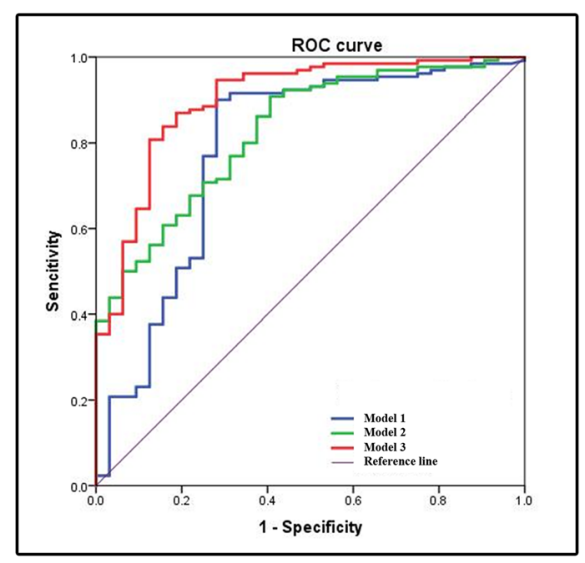

B

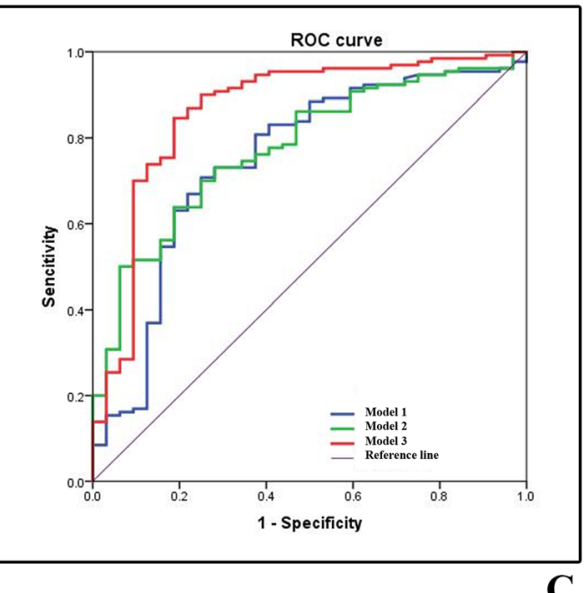

C

Fig. 4 Graphs show ROC curves for differentiation of ccRCCs and non-ccRCCs of Model 1, Model 2, and Model 3. A, Corticomedullary phase, CMP. B, Nephrographic phase, NP. C, Excretory phase, EP

accurate diagnosis. Like the study conducted by Young et al. [2] and others, the results showed high utility of enhancement values at multiphasic multidetector CT with regard to differentiation of ccRCC from pRCCs, chRCCs, and its benign mimics [2, 21, 31-37]. In our study, 43 enhancement values were concluded as nontexture features. To the best of our knowledge, Enhancement Change of aorta and Corrected CT values relative to renal cortex has not been reported yet. We found that the diagnostic performance of most enhancement values at CMP, NP and EP in differentiating ccRCC from nonccRCC was higher than lesion attenuation value did.

Although multiphasic CT has demonstrated useful in differentiation of RCC subtypes [8,38], various studies have been done on assessing the radiomics features for differentiating RCC subtypes. Feng et al. [16] collected 52 cases of fat-poor angiomyolipoma and ccRCC less than $4 \mathrm{~cm}$, and extracted CT texture features of the largest layer of tumor in NCP, CMP and NP. The results showed that the texture analysis method based on machine learning was conducive to the identification of the two, with an accuracy of $93.9 \%$ and AUC of 0.955 .

On this foundation, we have tried to move deeper. We extracted texture features and non-texture features to construct combined diagnosis models in order to improve the diagnostic performance, so as Jiule Ding did. Jiule Ding et al. [39] retrospectively extracted CT texture features of 114 ccRCCs in the CMP and NP, and established the non-texture features diagnosis model, texture features diagnosis model and combined diagnosis model. The results showed that the combined diagnosis model had the highest diagnostic efficiency in discrimination of the high from low grade ccRCC with the AUC values of 0.878 in the training cohort. The difference is that we did this research in another aspect. To the best of our knowledge, Texture-features based models with the nontexture features is rarely used in the identification of ccRCC and non-ccRCC. In the area of distinguishing ccRCC from non-ccRCC, Zhi-Cheng Li et al. [40] extracted the RFs of the 3D tumor in the CMP and NP, 
and concluded that the model in CMP achieved an AUC of 0.949 and an accuracy of $92.9 \%$. Although this article got better results, we covered more texture features in all NCP, CMP, NP and EP, and the results were more comprehensive and reliable. We also compared the diagnostic efficacy of three different diagnostic models in each phase, paving the way for further research and clinical application, which Zhi-Cheng Li did not do. Most importantly, Zhi-Cheng Li does not combine texture features with non-texture features, which is rarely used in previous study of identification ccRCC from non-ccRCC. We not only combined, but also mathematically improved the non-ccRCC feature algorithm to eliminate the individual differences.

Many researchers think the most reasonable performance metric is the accuracy, which refers to ratio between the number of correctly classified samples and the overall number of samples [41]. However, when the dataset is unbalanced, which is often seen in previous study, accuracy cannot be considered a reliable measure anymore, because it fail to consider the ratio between positive and negative elements [42-45]. Based on these reasons, this paper put forward MCC in addition to accuracy. To our knowledge, these parameters have been rarely mentioned in previous studies.

In this study, combined models of different phases were constructed, which indicates the value of texture features in the predictive model. Although Ding J et al. [39] declared that non-texture features had limited predictive value in discriminating the high nuclear grade from the low one. The Texture-score combined with non-texture features in our study improved the capacity of the prediction models for identify ccRCC. However, in other directions, the role of non-textural features needs to be further studied.

This study has the following innovations: First, we include entire 3D tumor tissue and four-phase images to analyze tumor texture features as fully as possible. Second, we applied the improved enhancement parameter based on the traditional $\mathrm{CT}$ value measurement method. This kind of non-texture features are rarely reported in previous studies, which is partly proposed for the first time, and we found that those non-texture features could identify ccRCC with higher diagnostic performance. Moreover, models combined Texture-score with non-texture features were also proposed in this research, which is rarely reported in literature. We also found that the combined diagnostic model was more effective than any single diagnostic model. Finally, $F_{1}$ and accuracy generate reliable results only when applied to balanced datasets, and produce misleading results when applied to imbalanced cases. MCC, instead, was used in our research to overcome the class imbalance issue. However, there were several limitations to our study. First, this study was based on single center. In hence, large, multicenter and prospective studies should be involved in future to validate the model. Another limitation of this study is the relatively unbalanced data set. Two-thirds of the data in this study consisted of ccRCC, while this was similar in size to previous studies regarding these relatively common renal lesions [46, 47]. In the process of data preprocessing, SMOTE was used to solve the problem of data imbalance as much as possible, but further analysis is still necessary to confirm our results. Besides, previous studies have shown the value of multiparametric MRI in ccRCC differentiation [7, 48]. Incorporating features derived from these MR modalities may potentially improve the model performance.

\section{Conclusion}

In conclusion, our results show that quantitative CT texture features along with non-texture features can be used to accurately differentiate ccRCC from non-ccRCC on CMP, NP, and EP. Our preliminary results reveal that the combination of texture feature model, and nontexture feature model constitute an optimal pipeline method preoperatively. However, this technique requires further validation on a larger scale prior to implementation into clinical practice.

\begin{abstract}
Abbreviations
ccRCC: clear cell renal cell carcinoma; non-ccRCC: non-clear cell renal cell carcinoma; NCP: Non-contrast Phase; CMP: Corticomedullary Phase; NP: Nephrographic Phase; EP: Excretory Phase; LASSO: Least Absolute Shrinkage and Selection Operator; AUC: Area under receiver operating characteristic curve; RCC: Renal cell carcinoma; pRCC: papillary renal cell carcinoma; chRCC: chromophobe renal cell carcinoma; CT: Computed Tomography; MR: Magnetic Resonance; RFs: Radiomic features; PACS: Picture archiving and communication system; SD: Standard deviation; FOV: Field of view; ROI: Region of interest; PPV: Positive predictive value; NPV: Negative predictive value; GLCM: Gray level cooccurrence matrix; GLRLM: Gray level run length matrix; GLSZM: Gray level size zone matrix; NGTDM: Neighbouring gray tone difference matrix; GLDM: Gray level dependence matrix;

Cl: Confidence interval; MCC: Matthews correlation coefficient
\end{abstract}

\section{Acknowledgements}

Not applicable.

\section{Authors' contributions}

Menglin Chen, Haijie Zhang and Ge Wen contributed to the design and implementation of the concept. Menglin Chen and Yuanmeng Yu

contributed in collecting and reviewing the data. Menglin Chen and Fu Yin contributed to the design and implementation of quantitative feature extraction models. Fu Yin contributed to the statistical analysis of the data. All authors contributed to the writing and reviewing of the paper. All authors read and approved the final manuscript.

\section{Funding}

This work was supported by the Scientific Research Foundation of Education Department of Yunnan Province(Grant number NO.2021 J0254), and Natural Science Foundation of Guangdong Province(Grant number NO.2020A1515010469).

\section{Availability of data and materials}

The datasets during and/or analyzed during the current study available from the corresponding author on reasonable request pending the approval of the institution and trial/study investigators who contributed to the dataset. 


\section{Declarations}

\section{Ethics approval and consent to participate}

The institutional research ethics board approved this retrospective single institution study and waived the requirement for informed consent.

\section{Consent for publication}

Not applicable.

\section{Competing interests}

None of the authors have any conflicts of interest to declare

\section{Author details}

${ }^{1}$ Medical Imaging teaching and research office, Nanfang hospital, Southern Medical University, No.1838 Guangzhoudadao Avenue north, Guangzhou 510515, Guangdong, China. ${ }^{2}$ Radiology department, The second affiliated hospital of Kunming medical university, No. 374 Dianmian Road, Kunming 650032, Yunnan, China. ${ }^{3}$ College of Electronics and Information Engineering, Shenzhen University, Shenzhen 518068, China. ${ }^{4}$ Department of MRI, The First People's Hospital of Yunnan Province, The Affiliated Hospital of Kunming University of Science and Technology, No. 157 Jinbi Road, Kunming 650032 Yunnan, China. ${ }^{5}$ Department of Radiology, Shenzhen Second People's Hospital, No.3002, West Sungang Road, Futian District, Shenzhen 518052, China.

Received: 23 July 2020 Accepted: 9 June 2021

Published online: 23 June 2021

\section{References}

1. Cheville JC, Lohse CM, Zincke H, Weaver AL, Blute ML. Comparisons of Outcome and Prognostic Features Among Histologic Subtypes of Renal Cell Carcinoma. Am J Surg Pathol. 2003;27(5):612-24

2. Young JR, Margolis D, Sauk S, Pantuck AJ, Sayre J, Raman SS. Clear Cell Renal Cell Carcinoma: Discrimination From Other Renal Cell Carcinoma Subtypes and Oncocytoma at Multiphasic Multidetector Ct. Radiology. 2013; 267(2):444-53.

3. Armstrong AJ, Halabi S, Eisen T, Broderick S, Stadler WM, Jones RJ, et al. Everolimus Versus Sunitinib for Patients with Metastatic Non-Clear Cell Renal Cell Carcinoma (Aspen): A Multicentre, Open-Label, Randomised Phase 2 Trial. Lancet Oncol. 2016;17(3):378-88.

4. Fernandez-Pello S, Hofmann F, Tahbaz R, Marconi L, Lam TB, Albiges L, et al. A Systematic Review and Meta-Analysis Comparing the Effectiveness and Adverse Effects of Different Systemic Treatments for Non-Clear Cell Renal Cell Carcinoma. Eur Urol. 2017;71(3):426-36.

5. Mileto A, Nelson RC, Paulson EK, Marin D. Dual-energy Mdct for imaging the renal mass. AJR Am J Roentgenol. 2015;204(6):W640-7. https://doi.org/1 0.2214/AJR.14.14094

6. Mileto A, Marin D, Alfaro-Cordoba M, Ramirez-Giraldo JC, Eusemann CD, Scribano E, et al. lodine Quantification to Distinguish Clear Cell From Papillary Renal Cell Carcinoma at Dual-Energy Multidetector Ct: A Multireader Diagnostic Performance Study. Radiology. 2014;273(3):813-20.

7. Hotker AM, Mazaheri Y, Wibmer A, Karlo CA, Zheng J, Moskowitz CS, et al. Differentiation of clear cell renal cell carcinoma from other renal cortical tumors by use of a Quantitative multiparametric Mri approach. AJR Am J Roentgenol. 2017;208(3):W85-91. https://doi.org/10.2214/AJR.16.16652.

8. Lopes VC, Parada VC, TJ DJ, Chatterjee A, Casalino DD, Horowitz JM, et al. Differentiation of Solid Renal Tumors with Multiparametric Mr Imaging. Radiographics. 2017;37(7):2026-42.

9. Lambin P, Leijenaar R, Deist TM, Peerlings J, de Jong E, van Timmeren J, et al. Radiomics: The Bridge Between Medical Imaging and Personalized Medicine. Nat Rev Clin Oncol. 2017;14(12):749-62.

10. Gillies RJ, Kinahan PE, Hricak H. Radiomics: Images are More than Pictures, they are Data. Radiology. 2016;278(2):563-77.

11. van Griethuysen J, Fedorov A, Parmar C, Hosny A, Aucoin N, Narayan V, et al. Computational Radiomics System to Decode the Radiographic Phenotype. Cancer Res. 2017:77(21):e104-7.

12. Aerts HJ, Velazquez ER, Leijenaar RT, Parmar C, Grossmann P, Carvalho S, et al. Decoding Tumour Phenotype by Noninvasive Imaging Using a Quantitative Radiomics Approach. Nat Commun. 2014;5:4006.
13. Huang Y, Liang C, He L, Tian J, Liang C, Chen X, et al. Development and Validation of a Radiomics Nomogram for Preoperative Prediction of Lymph Node Metastasis in Colorectal Cancer. J Clin Oncol. 2016;34(18):2157-64.

14. Hodgdon T, Mclnnes MD, Schieda N, Flood TA, Lamb L, Thornhill RE. Can Quantitative Ct Texture Analysis be Used to Differentiate Fat-Poor Renal Angiomyolipoma From Renal Cell Carcinoma On Unenhanced Ct Images? Radiology. 2015;276(3):787-96.

15. Takahashi N, Takeuchi M, Sasaguri K, Leng S, Froemming A, Kawashima A. Ct Negative Attenuation Pixel Distribution and Texture Analysis for Detection of Fat in Small Angiomyolipoma On Unenhanced Ct. Abdom Radiol (NY). 2016;41(6):1142-51.

16. Feng Z, Rong P, Cao P, Zhou Q, Zhu W, Yan Z, et al. Machine LearningBased Quantitative Texture Analysis of Ct Images of Small Renal Masses: Differentiation of Angiomyolipoma without Visible Fat From Renal Cell Carcinoma. Eur Radiol. 2018;28(4):1625-33.

17. Zhang GM, Shi B, Sun H, Jin ZY, Xue HD. Differentiating Pheochromocytoma From Lipid-Poor Adrenocortical Adenoma by Ct Texture Analysis: Feasibility Study. Abdom Radiol (NY). 2017;42(9):2305-13.

18. Schieda N, Lim RS, Krishna S, Mclnnes MDF, Flood TA, Thornhill RE. Diagnostic accuracy of unenhanced CT analysis to differentiate low-grade from high-grade chromophobe renal cell Eur Radiolcarcinoma. AJR Am J Roentgenol. 2018:1-9. https://doi.org/10.2214/AJR.17.18874.

19. Collewet G, Strzelecki M, Mariette F. Influence of MRI acquisition protocols and image intensity normalization methods on texture classification. Magn Reson Imaging. 2004;22:81-91. https://doi.org/10.1016/j.mri.2003.09.001.

20. Shafiq-ul-Hassan M, Zhang GG, Latifi K, Ullah G, Hunt DC, Balagurunathan Y, et al. Intrinsic dependencies of CT radiomic features on voxel size and number of gray levels. Med Phys. 2017;44:1050-62. https://doi.org/10.1002/ mp.12123.

21. Ishigami K, Pakalniskis MG, Leite LV, Lee DK, Holanda DG, Rajput M. Characterization of Renal Cell Carcinoma, Oncocytoma, and Lipid-Poor Angiomyolipoma by Unenhanced, Nephrographic, and Delayed Phase Contrast-Enhanced Computed Tomography. Clin Imaging. 2015;39(1):76-84.

22. Udare A, Walker D, Krishna S, Chatelain R, Mclnnes MD, Flood TA, et al. Characterization of Clear Cell Renal Cell Carcinoma and Other Renal Tumors: Evaluation of Dual-Energy CT Using Material-Specific lodine and Fat Imaging. Eur Radiol. 2020;30(4):2091-102.

23. Kocak B, Ates E, Durmaz ES, Ulusan MB, Kilickesmez O. Influence of Segmentation Margin On Machine Learning-Based High-Dimensional Quantitative Ct Texture Analysis: A Reproducibility Study On Renal Clear Cell Carcinomas. Eur Radiol. 2019;29(9):4765-75.

24. Chawla NV, Bowyer KW, Hall LO, et al. SMOTE: synthetic minority oversampling technique. J Artif Intell Res. 2011;16:321-57.

25. Fehr D, Veeraraghavan H, Wibmer A, Gondo T, Matsumoto K, Vargas HA et al. Automatic classification of prostate cancer Gleason scores from multiparametric magnetic resonance images. Proc Natl Acad Sci. 2015; 112(46):E6265-73. https://doi.org/10.1073/pnas.1505935112.

26. Zhang Y, Oikonomou A, Wong A, Haider MA, Khalvati F. Radiomics-based prognosis analysis for non-small cell lung Cancer. Sci Rep. 2017;7(1):46349. https://doi.org/10.1038/srep46349.

27. Baldi $P$, Brunak S, Chauvin $Y$, Andersen CA, Nielsen H. Assessing the accuracy of prediction algorithms for classification: an overview. Bioinformatics. 2000;16(5):412-24. https://doi.org/10.1093/bioinformatics/16. 5.412

28. Gorodkin J. Comparing two K-category assignments by a K-category correlation coefficient. Comput Biol Chem. 2004;28(5-6):367-74. https://doi. org/10.1016/j.compbiolchem.2004.09.006.

29. Zhang J, Lefkowitz RA, Ishill NM, Wang L, Moskowitz CS, Russo P, et al. Solid Renal Cortical Tumors: Differentiation with Ct. Radiology. 2007;244(2):494504.

30. Banno T, Takagi T, Kondo T, Yoshida K, lizuka J, Okumi M, et al. Computed Tomography Imaging Characteristics of Clear Cell Papillary Renal Cell Carcinoma. Int Braz J Urol. 2020;46(1):26-33.

31. Kim SH, Kim CS, Kim MJ, Cho JY, Cho SH. Differentiation of clear cell renal cell carcinoma from other subtypes and fat-poor Angiomyolipoma by use of Quantitative enhancement measurement during three-phase Mdct. AJR Am J Roentgenol. 2016;206(1):W21-8. https://doi.org/10.2214/AJR.15.14666.

32. Coy H, Young JR, Douek ML, Brown MS, Sayre J, Raman SS. Quantitative Computer-Aided Diagnostic Algorithm for Automated Detection of Peak Lesion Attenuation in Differentiating Clear Cell From Papillary and Chromophobe Renal Cell Carcinoma, Oncocytoma, and Fat-Poor 
Angiomyolipoma On Multiphasic Multidetector Computed Tomography. Abdom Radiol (NY). 2017;42(7):1919-28.

33. Lee-Felker SA, Felker ER, Tan N, Margolis DJ, Young JR, Sayre J, et al. Qualitative and Quantitative Mdct features for differentiating clear cell renal cell carcinoma from other solid renal cortical masses. AJR Am J Roentgenol. 2014;203(5):W516-24. https://doi.org/10.2214/AJR.14.12460.

34. Yang CW, Shen SH, Chang YH, Chung HJ, Wang JH, Lin AT, et al. Are there useful $\mathrm{Ct}$ features to differentiate renal cell carcinoma from lipid-poor renal Angiomyolipoma? AJR Am J Roentgenol. 2013;201(5):1017-28. https://doi. org/10.2214/AJR.12.10204.

35. Bata P, Gyebnar J, Tarnoki DL, Tarnoki AD, Kekesi D, Szendroi A, et al. Clear Cell Renal Cell Carcinoma and Papillary Renal Cell Carcinoma: Differentiation of Distinct Histological Types with Multiphase Ct. Diagn Interv Radiol. 2013; 19(5):387-92.

36. Pierorazio PM, Hyams ES, Tsai S, Feng Z, Trock BJ, Mullins JK, et al. Multiphasic Enhancement Patterns of Small Renal Masses $(\& L \mathrm{~L} ; /=4 \mathrm{Cm})$ On Preoperative Computed Tomography: Utility for Distinguishing Subtypes of Renal Cell Carcinoma, Angiomyolipoma, and Oncocytoma. UROLOGY. 2013; 81(6):1265-71.

37. Millet I, Doyon FC, Hoa D, Thuret R, Merigeaud S, Serre I, et al. Characterization of small solid renal lesions: can benign and malignant tumors be differentiated with Ct? AJR Am J Roentgenol. 2011;197(4):887-96. https://doi.org/10.2214/AJR.10.6276.

38. Davenport MS, Chandarana H, Curci NE, Doshi A, Kaffenberger SD, Pedrosa I. et al. Society of Abdominal Radiology Disease-Focused Panel On Renal Cell Carcinoma: Update On Past, Current, and Future Goals. Abdom Radiol (NY). 2018;43(9):2213-20.

39. Ding J, Xing Z, Jiang Z, Chen J, Pan L, Qiu J, et al. Ct-Based Radiomic Model Predicts High Grade of Clear Cell Renal Cell Carcinoma. Eur J Radiol. 2018; 103:51-6.

40. Li ZC, Zhai G, Zhang J, Wang Z, Liu G, Wu GY, et al. Differentiation of clear cell and non-clear cell renal cell carcinomas by all-relevant radiomics features from multiphase CT: a VHL mutation perspective. Eur Radiol. 2019; 29(8):3996-4007. https://doi.org/10.1007/s00330-018-5872-6.

41. Wang L, Chu F, Xie W. Accurate cancer classification using expressions of very few genes. IEEE/ACM Trans Comput Biol Bioinform. 2007;4(1):40-53. https://doi.org/10.1109/TCBB.2007.1006.

42. Sokolova M, Japkowicz N, Szpakowicz S. Beyond accuracy, F-score and ROC: a family of discriminant measures for performance evaluation. In: Proceedings of Advances in Artificial Intelligence (Al 2006), Lecture Notesin Computer Science, vol. 4304. Heidelberg: Springer; 2006. p. 1015-21.

43. Gu Q, Zhu L, Cai Z. Evaluation measures of the classification performance of imbalanced data sets. In: Proceedings of ISICA 2009 the 4th international symposium on computational intelligence and intelligent systems, Communications in Computer and Information Science, vol. 51. Heidelberg: Springer; 2009. p. 461-71.

44. Bekkar M, Djemaa HK, Alitouche TA. Evaluation measures for modelsassessment over imbalanced data sets. J Inf Eng Appl. 2013;3(10):2738.

45. Akosa JS. Predictive accuracy: a misleading performance measure for highly imbalanced data. In: Proceedings of the SAS global forum 2017 conference. Cary: SAS Institute Inc.; 2017. p. 942-2017.

46. Zhang YY, Luo S, Liu Y, Xu RT. Angiomyolipoma with minimal fat: differentiation from papillary renal cell carcinoma by helical CT. Clin Radiol. 2013;68(4):365-70. https://doi.org/10.1016/j.crad.2012.08.028.

47. Hodgdon T, McInnes MDF, Schieda N, Flood TA, Lamb L, Thornhill RE. Can Quantitative $C T$ texture analysis be used to differentiate fat-poor renal Angiomyolipoma from renal cell carcinoma on unenhanced CT images? Radiology. 2015;276(3):787-96. https://doi.org/10.1148/radiol.2015142215.

48. Gaing B, Sigmund EE, Huang WC, Babb JS, Parikh NS, Stoffel D, et al. Subtype Differentiation of Renal Tumors Using Voxel-Based Histogram Analysis of Intravoxel Incoherent Motion Parameters. Investig Radiol. 2015; 50(3):144-52.

\section{Publisher's Note}

Springer Nature remains neutral with regard to jurisdictional claims in published maps and institutional affiliations.

Ready to submit your research? Choose BMC and benefit from:

- fast, convenient online submission

- thorough peer review by experienced researchers in your field

- rapid publication on acceptance

- support for research data, including large and complex data types

- gold Open Access which fosters wider collaboration and increased citations

- maximum visibility for your research: over $100 \mathrm{M}$ website views per year

At BMC, research is always in progress.

Learn more biomedcentral.com/submissions 